\title{
Percepção dos homens colaboradores de uma instituição de ensino superior sobre os seus cuidados a saúde
}

Perception of men who collaborate in a higher education institution on your health care Percepción de hombres colaboradores de uma institución de educación superior sobre su atención médica

\section{Resumo}

Na tentativa de enriquecer conteúdos de educação em saúde observou-se os desafios encontrados pelos homens frente as suas necessidades de saúde. O reconhecimento de facilitar e ampliar o acesso da população masculina aos serviços de saúde é uma iniciativa que resulta da observação de que os agravos do sexo masculino que são considerados um problema de saúde pública. Com o objetivo de refletir a percepção dos homens colaboradores de uma universidade brasileira acerca dos seus cuidados a saúde. Trata-se de um estudo qualitativo, do tipo descritivo e exploratório, por meio de grupo focal baseada na comunicação e na interação, com um grupo de 06 pessoas e aproximadamente 70 minutos de coleta, com a finalidade da abordagem da enfermagem na saúde do homem, os dados foram analisados por meio da análise de conteúdo. Contudo, o cuidado que muitas vezes não é visto como uma cultura masculina, o homem necessita da prática das políticas de saúde que tem por objetivo a promoção e prevenção de uma atenção básica mais atuante. Cabe ressaltar que a criação do Politica Nacional de Atenção Integral a Saúde do Homem, como um grande passo para a atuação na saúde do homem, ainda não se tem percebido mudanças efetivas no Sistema Único de Saúde, mas se almeja um futuro promissor na área.

Palavras-chave: Atenção Primaria à Saúde; Educação em Saúde; Saúde do Homem.

\begin{abstract}
In an attempt to enrich health education content, the challenges faced by men in relation to their health needs were observed. The recognition of facilitating and expanding the access of the male population to health services is an initiative that results from the observation that male injuries are considered a public health problem. In order to reflect the perception of male collaborators at a Brazilian university about their health care. This is a qualitative, descriptive and exploratory study, through a focus group based on communication and interaction, with a group of 06 people and approximately 70 minutes of collection, with the purpose of addressing nursing in men's health, the data were analyzed through content analysis. However, care that is often not seen as a male culture, men need the practice of health policies that aim to promote and prevent more active primary care. It is worth mentioning that the creation of the National Policy for Integral Attention to Men's Health, as a major step towards acting in men's health, has not yet noticed effective changes in the Unified Health System, but it looks forward to a promising future in the area.
\end{abstract}

Keywords: Primary Health Care; Health education; Men's Health.

\section{Resumen}

En un intento por enriquecer el contenido de la educación en salud, se observaron los desafíos que enfrentan los hombres en relación con sus necesidades de salud. El reconocimiento de facilitar y ampliar el acceso de la población masculina a los servicios de salud es una iniciativa que resulta de la observación de que las lesiones masculinas son consideradas un problema de salud pública. Con el fin de reflejar la percepción de los colaboradores masculinos de una universidad brasileña sobre su 
cuidado de la salud. Se trata de un estudio cualitativo, descriptivo y exploratorio, mediante un grupo focal basado en la comunicación e interacción, con un grupo de 06 personas y aproximadamente 70 minutos de recolección, con el propósito de abordar la enfermería en la salud del hombre, Los datos fueron analizados a través de contenido análisis. Sin embargo, una atención que a menudo no se ve como una cultura masculina, los hombres necesitan la práctica de políticas de salud que tengan como objetivo promover y prevenir una atención primaria más activa. Cabe mencionar que la creación de la Política Nacional de Atención Integral a la Salud del Hombre, como un gran paso para actuar en la salud del hombre, aún no ha notado cambios efectivos en el Sistema Único de Salud, pero apunta a un futuro promisorio en el área.

Palabras clave: Atención Primaria de Salud; Educación para la salud; Salud de los hombres.

\section{Introdução}

Desde os primórdios da humanidade, a masculinidade foi dirigida por um processo histórico, cujo questões culturais estabelecia uma hierarquia entre os sexos, então o homem apresentava consigo uma supremacia, percebendo-se como ser invulnerável, onde a força, trabalho, liderança e a virilidade tornaram-se fatores que o levaram a desvalorização do seu autocuidado (Cavalcanti et al., 2014).

Do ponto de vista organizacional o modo como o sistema de saúde no Brasil vem sendo praticado mostra que os homens são menos prioritários em relação aos demais grupos populacionais, no Sistema Único de Saúde (SUS) estão os serviços condizentes aos atendimentos a população masculina, dentre os quais se encontram menos procurados, o que inviabiliza uma escuta qualificada e possível centralização das necessidades de saúde para este grupo (Silva et al., 2012).

Nos costumes masculinos hegemônico, ser homem está ligado à invulnerabilidade, por se sentirem envergonhados em procurar serviços de saúde, pois para os mesmos, seria expressão de fraqueza perante os profissionais, essas manifestações culturais masculinas acabam tornando-se um obstáculo a ser vencido e dificultando cada vez mais a participação da população masculina nos serviços de saúde (Cavalcanti et al., 2014).

Em contrapartida, medidas promovidas pelo Ministério da Saúde (MS) a fim de minimizar essa disparidade e promover maior equidade entre os sexos vêm ganhando espaço no Brasil. Com isso a portaria $\mathrm{n}^{\circ} 1.944$ de 27 de agosto de 2009, instituiu em seu âmbito a Política Nacional de Atenção Integral à Saúde do Homem (PNAISH), visto a necessidade dos cuidados com a saúde da população masculina (Brasil, 2009).

A proposta torna-se desafiadora, pois os homens não têm conceito de valorização e cuidado do corpo tratando a saúde como questões sociais. Por ser pouco abordada e discutida torna-se evidente o prejuízo existente ao sexo masculino, pois grande parte das ações são voltadas para saúde da mulher, criança e idoso dificultando o acesso à saúde do homem aos serviços de saúde, sendo considerados mais vulneráveis (Silva et al., 2013).

É valido destacar que através de formas preventivas no cuidado a saúde, muitas doenças podem ser evitadas, mas a notória resistência masculina quando relacionada a prevenção, enfraquece a promoção da saúde, pois tendem a procurar atendimento a partir do momento que a patologia já apresenta estágios avançados, priorizar ações de atenção secundária ou terciaria (Silva et al., 2013).

Para beneficiar o homem implementando serviços que atendam às suas necessidades, é indispensável uma estrutura de base acerca do acolhimento na finalidade do cuidar, de se ter uma atenção voltada a saúde masculina, bem como estratégias educativas que possam auxiliar no acesso dessa população nas instituições afim de realizar exames preventivos com a maior agilidade possível (Silva et al., 2015).

Nestes casos, os profissionais de saúde necessitam da aquisição de diferentes formas de pensar, quebrando crenças e valores para incorporar novos conceitos da saúde masculina, realizando ações de orientações, com a finalidade de que o indivíduo não adoeça, e desta forma, possa ter uma melhor qualidade de vida (Silva et al., 2013).

Contudo, deve-se ter ações direcionadas à assistência interdisciplinar de modo a articular práticas assistenciais curativas e preventivas. Se faz necessário refletir quanto a percepção dos homens colaboradores da instituição de ensino sobre 
seus cuidados a saúde, para tal enfrentamento, se torna relevante desenvolver uma visão qualificada o que fara uma assistência mais eficiente, para a redução do número de patologias e de agravos na população masculina.

\section{Metodologia}

Trata-se de um estudo qualitativo realizado em uma Instituição de Ensino Superior localizada no agreste Pernambucano no mês de novembro de 2018 com homens colaboradores da instituição.

Como critérios de inclusão para participar do estudo os colaboradores precisaram estar vinculados a instituição por um período mínimo de 2 anos, possuir curso superior completo e aceitarem participar do estudo. Não foram incluídos colaboradores docentes e os que estivessem afastados por problemas de saúde ou de licença. A coleta de dados foi realizada através de um grupo focal e para a análise foi empregada à técnica de análise de conteúdo proposta por Bardin (2009).

Participaram desse estudo, 6 homens em um grupo focal que teve duração de 1 hora e 07 minutos. Para a seleção foi convidado todos os colaboradores que atenderam aos critérios de inclusão ao final do expediente de trabalho, porém apenas 7 homens aceitaram participar sendo 1 excluído por não atender aos critérios do estudo. Com a finalidade de manter o sigilo dos sujeitos entrevistados, foi atribuído um número sequencial para identificação do material referente a cada estudante, identificando-os como E1, E2, E3, E4, E5 e E6.

O estudo atende aos preceitos éticos e legais da resolução no. 466/12 do Conselho Nacional de Saúde e foi aprovado pelo Comitê de Ética e Pesquisa sob o número 2.956.524.

\section{Resultados e Discussão}

Após a transcrição, leitura e estudo das falas pelos pesquisadores, foram definidas duas categorias distintas: 1) A percepção do homem sobre sua busca ao serviço de saúde; 2) O (Des) Conhecimento do homem quanto aos serviços de saúde.

\section{Categoria I - A percepção do homem sobre sua busca ao serviço de saúde}

É inegável as marcas na cultura histórica da humanidade que enfatiza a soberania masculina, o que passa por despercebido é que essa ideia, é apenas um modo de camuflar suas fragilidades. Em geral, os homens padecem mais de condições severas e crônicas de saúde do que as mulheres.

Um dos fatores que se conecta a essa problemática é a dificuldade existente neste grupo, em reconhecer suas próprias necessidades de saúde, abdicando a possibilidade de adoecer, mantendo até os dias atuais a questão cultural da invulnerabilidade masculina, tendo seu papel social como provedor e herói.

Considerando essa representação do "ser homem" na sociedade e o seu imaginário, é pertinente fazer um alusão a um super-herói criado pela dupla de autores de quadrinhos Joe Shuster e Jerry Siege: O SUPERMAN, por se tratar de um ser viril, forte, trabalhador e quase invulnerável ainda é inspiração de crianças, adolescentes e adultos de como um homem deve ser. Porém essa construção social de masculinidade que ajuda a construir caráter e valores positivos também cria um homem quase invulnerável em questões de saúde, fato que em nossa realidade associada as limitações do serviço de saúde para atender ao público masculino traz condições que levam o homem a uma cultura de desvalorização do seu autocuidado.

A partir da discussão dos colaboradores, foi evidenciado que seus comportamentos de autocuidado têm influências culturais muito fortes envolvendo regiões geográficas e até representações etárias para o público masculino, como pode-se perceber nas seguintes falas: 
E1: só vai em busca de tratamento quando tá em estágio avançando, ou quando tem alguma doença, que acomete alguma doença, algo grave e daí procura-se né, [...] caso não sempre vai levando com a barriga isso é sempre a cultura do homem, principalmente o homem nordestino né.

E2: ou tá meio fora da curva que eu estava, a crise dos 30 tá chegando eu já fiz checkup geral, eu entrei na academia (risos), já tô dando uma cuidada já pra...

E5: [...] essa questão da cultura ela é muito intensificada principalmente aqui em nossa região né, de deixar pra último caso, pra último momento [...]

Diante das falas Sousa et al. (2016), em seu estudo sobre a construção social da masculinidade em defesa influenciada por aspectos culturais na masculinidade, fortalece a ideia de que, geralmente, nas mais variadas culturas, a educação dos meninos acompanha padrões de aversão entre os sexos, desta forma os homens são estimulados a tornar-se visível a sua virilidade por meio do abandono de comportamentos tidos como femininos para se constituírem como homens, assim o padrão de masculinidade é idealizado pelos meninos/homens não pelo incessante desejo de serem viris, mas pelo receio de serem observados como pouco viris ou afeminados.

É valido destacar Lopes, Sardagna e Lervolino (2017), em seu estudo referente aos motivos que leva os homens ao serviço de pronto atendimento refere que o autocuidado é justificado pelos homens para a não procura aos serviços de atendimento primário, porém isto acaba ocasionando em uma maior vulnerabilidade da população masculina à saúde. Visto que os homens afirmam está realizando o autocuidado da forma correta, entretanto, alguns não sabe realmente o que seria.

Barreto, Arruda e Marcon (2015), em seu estudo a respeito de como os homens avaliam os serviços de saúde acrescenta a população masculina faz uso da automedicação muitas vezes, quando considera o problema pode ser resolvido em casa mesmo, sem necessitar de ir a Unidade Básica de Saúde - UBS para atendimento dos profissionais. Contudo, acaba mostrando a falha que possui no seu autocuidado, pois o uso inadequado de medicamentos pode ocasionar sérios problemas a saúde, além de dificulta o trabalho dos profissionais.

A proposta da inclusão da masculinidade em ações de saúde torna-se trabalhosa, por não terem o reconhecimento do cuidado e valorização do corpo no propósito da saúde como questões sociais. De certa forma existe um preconceito evidente em relação ao sexo masculino, pois é pouca abordada, além de se ter uma carência de conhecimentos nas campanhas de saúde pública que não são voltadas a este segmento da população. Isto pode ser observado nas seguintes falas:

E1: Embora saibamos que não é cultura do homem se cuidar, sempre tem aquela cultura de deixar para depois, $e$ isso é ser coisa de mulher [...]

E5: [...]por ser homem deixar mais pra frente, não, não to doente, não, não preciso é sempre muito negativo essa questão ai do auto cuidado.

E3: Digamos que a gente tem uma resistência a aceitar a pratica de se cuidar.

E4: Ou deixar quando já tá né, caindo aos pedaços que tem que ir pro médico, ta com uma dor de cabeça ou coisa mais pior, então, fica essa questão de deixar para última hora.

Esses dados parecem estar de acordo com achados do estudo de Oliveira et al., (2013) sobre a busca do homem por atendimento na atenção básica, entende-se que ser homem é representar um perfil repleto de acepções e sentidos, e a demonstração de sua masculinidade é relacionada, ao exemplo de masculinidade hegemônica, onde o homem deve ser forte e 
invulnerável. Essas características fazem com que a maioria dos homens adotem uma postura de baixo autocuidado se afastando dos serviços de saúde, mais precisamente da atenção primária.

Os estudos de Vieira et al., (2013) sobre os motivos que levam a não procura de atendimentos por parte dos homens nas unidades básicas de saúde, corroboram com os aspectos em questão que vários determinantes são explicados pelo os homens, de não buscar atendimento nos serviços de saúde, dentre estes, o medo de descobrir algo mais grave, a demora no atendimento, a vergonha de se expor para os profissionais, o não reconhecimento como alvo frágil de atendimento, estereótipos de gênero que atrapalham o autocuidado, questões socioculturais, além do horário de funcionamento dos serviços de saúde que coincidem com o horário de trabalho.

No seu estudo sobre as experiências dos homens em um determinado centro de saúde Bertolinil e Simonettil (2014) buscaram compreender os diversos motivos dos homens buscarem os serviços de saúde já com doenças instaladas, é perceptível a valorização das mulheres em detrimento dos homens, já que os mesmos seriam mais predispostos ás praticas curativas e menos preparado para a prevenção, deste modo, a invisibilidade masculina demonstra que os homens não cuidam de sim, portanto não procuram os serviços ofertados ou o fazem de forma menos autênticas.

Ficou evidente a afirmação dos entrevistados que expõe as raras circunstâncias na qual os homens procuram assistência, habitualmente isto acontece em apenas duas situações, quando a dor se torna intolerável e/ou quando seu quadro impede que o mesmo trabalhe. De acordo com Vieira et al., (2013) assim como complementam outros estudos em que ocasiões nas quais são analisadas sem risco, os homens procuram fazer uso de meios alternativos, como por exemplo à automedicação.

Segundo o Ministério da Saúde (2009), indivíduos do sexo masculino orgulhando-se de sua invulnerabilidade evitam procura os serviços de saúde, seja através das unidades de saúde pública ou consultórios, possibilitando assim maior agravo nos casos de doença e consequentemente causando mais gastos para o sistema de saúde e concomitantemente para si, tendo em vista que os atendimentos prestados são nos níveis mais avançados como o secundário e terciários.

Sabe-se que o melhor local para estimular essa população masculina a adquirir hábitos preventivos é através da atenção primaria ofertada pelas UBS, que são consideradas como porta de entrada para o Sistema Único de Saúde - SUS, entretanto, observa-se que a presença de homens nesses serviços a saúde é menor do que as mulheres.

Com a análise do estudo observamos que os homens optam por dar entrada nos serviços de saúde por meio da atenção secundária e terciária, trazendo na maioria das vezes consequências no agravo da morbidade devido à demora pela à procura do serviço de saúde, como pode ser observado nas seguintes falas:

E1: Eu prefiro assistência especializada consultório. Pela praticidade, liga, faz o agendamento, vai...

E4: Eu também, você ter a certeza que você será atendido, naquele dia, naquele horário, coisa que se você for para o postinho de saúde do bairro, tanto faz ter como não ter, e depois você vai ter que marcar, então não é um imediatismo como toda vez a gente pensa né, realmente é na urgência, e naquela hora que você tá precisando, só encontra isso quando você vai para rede pública.

E2: Eu também [...] eu já acompanhei minha mãe já, pra poder fazer... para marcar consulta já, sei que existe também o agendamento para poder fazer, só que aí é aquela questão que você precisa se deslocar, não existir um mecanismo para agendamento tão simples[...]

Conforme Lopes, Sardagna e Lervolino (2017), nas Unidades Básica de Saúde - UBS os problemas no funcionamento são pouco decisivos, as dificuldades no funcionamento pouco resolutivo, tendo em consideração as filas de espera para 
atendimento, podendo ocasionar a perca de um dia de trabalho sem que tenha a certeza que o problema possa ser resolvido, tal razão, frequentemente, é que colabora para evasão dos homens que buscam por atendimento na atenção primaria.

Em seus estudos sobre possibilidades e limites referente ao atendimento noturno nas unidades básicas Cordeiro et al., (2014), observou que a população masculina nega suas necessidades de se saúde, assim sendo, procuram menos os serviços comparado com as mulheres e quando faz uso do sistema de saúde, já escolhem os serviços de atenção secundaria e terciaria ou especializado. Contudo, muitos acabam optando por buscar atendimento nos serviços de pronto atendimento.

Deste modo Cordeiro et, al. (2014), traz que é substancial a atuação dos profissionais de saúde, em atuações que busquem além da vacinação, esclarecer aos homens as dúvidas e instigar sobre a forma correta de autocuidado, como também mostra e estimular eles sobre a importância da participação deste tipo de público nas unidades de saúde.

\section{Categoria II - O (Des)conhecimento do homem quanto aos serviços de saúde}

No ano de 2009, foi fundada a Política Nacional de Atenção Integral a Saúde do Homem - PNAISH pelo o Ministério da Saúde, cuja o objetivo é de promover atividades voltada para os homens, proporcionando melhorias nas condições da população masculina brasileira e ampliando a entrada na atenção primaria. Assim sendo, a política tem a finalidade educativa e descreve estratégias para os homens, além de promover a promoção, prevenção, tratamento e reabilitação à saúde.

Entretanto, apesar de nove anos da criação da PNAISH, pouco são os homens que sabem a respeito do que é a política, e qual seu objetivo, como podemos observa na fala dos participantes:

\section{E1: Não conheço. \\ E2: Não conheço. \\ E3: Não conheço. \\ E4: Não conheço.}

E5: Eu já, mais eu creio.... foi bem recente a um ano, que eu tenho um amigo, que ele se formou aqui, e uma da... dos projetos dele foi referente a saúde do homem, mas eu não sabia até o momento que tinha essa [...].

O Ministério da Saúde (2009), agrupado com as esferas estaduais e municipais que compõe o SUS, entendeu que para alcançar mais rápido melhor indicador de qualidade de vida e longos padrões de vida é primordial desenvolver cuidados específicos para o homem jovens e adultos. Se trata de chamar atenção dos homens para o cuidado e proporcionar serviços de saúde que facilitem o embate dos agravos que são específicos da masculinidade.

Com base no estudo de Moreira e Carvalho (2016), sobre estratégias utilizadas por enfermeiros nas Unidades de Saúde da Família - USF, que tem como objetivo principal da PNAISH causar modificações impactantes sobre a saúde dos usuários masculinos, tornando-os figurante de sua própria saúde, a política visa implantar nos serviços de saúde, uma rede de Atenção Integral à Saúde do Homem - AISH, a qual garante linhas de cuidado, qualificando o profissional para uma melhoria na qualidade da assistência.

De acordo com o Ministério da Saúde (2009), a PNAISH junto com a Política Nacional de Atenção Básica (PNAB), promovem ações que contribuam significativamente para o entendimento da realidade masculina, com suas estratégias de humanização, no intuito do fortalecimento das ações e dos serviços disponibilizados para população, a política traduz uma inquietação da sociedade ao identificar que os agravos masculinos integram verdadeiros problemas de saúde pública. 
A saúde do homem pode ter avançado ao longo dos anos, porém não possui o mesmo destaque nas ações das políticas públicas como dos demais grupos de mulheres, criança e idosos. Mas é inegável a importância do estimulo do homem a procura do cuidado da sua saúde.

Deve ser objeto de investigação e reconhecimento social, onde existe a necessidade de aumentar o nível de conscientização entre os homens quanto a prevenção e promoção ofertada pelo serviço primário de saúde. Campanhas como o novembro azul surge para incentivando a procura do homem ao serviço de saúde para garantir que o homem crie o habito dos cuidados, utilizando diferentes meios para alcança-los através de ações educativas.

No entanto o conhecimento desses homens quanto as ações de saúde são desconhecidas restringindo apenas em uma única temática: o câncer de próstata, como podemos ver a seguir:

\section{E5: A questão da campanha do exame de próstata, é a que eu conheço [...].}

E4: [...] o novembro é para o homem da próstata, mas eu acho que não se resume só a isso [...] mas eu acho que por trás tem outros cuidados, como agente discutiu e deve ser visto para tirar de fato só esse foco, porque tipo se for só próstata a gente... no fim... a gente não tem esse cuidado.

E2: Restringe a uma faixa etária e a gente acaba não se enquadrando, e acaba nem procurando.

No seu estudo sobre o adoecimento dos homens, Modena (2013) afirma que, embora esse grupo populacional apresenta noções significativas sobre o câncer de próstata e reconhecimento a respeito das formas de diagnóstico precoce, verifica-se a pouca adesão as medidas preventivas, principalmente a respeito da difícil inclusão de exames anuais preventivos na rotina.

Para Vieira et al., (2013) os profissionais de saúde necessitam utilizar conduta de acolhimento de forma diferenciada com os homens que buscam os serviços de saúde, visto que, cada um possuem suas particularidades, este tipo de público precisa se sentir à vontade e se reconhecer, bem como os mesmos precisam modificar sua compreensão em relação com o cuidado com sua saúde.

Corroborando com o estudo de Silva et al., (2013), sobre métodos preventivos a saúde do homem, se torna necessário aumentar o nível de conscientização, quanto à prevenção, promoção e adesão do homem ao serviço primário de saúde, e o conhecimento da população masculina sobre os fatores de risco das doenças acometidas.

De conformidade com Moreira e Carvalho (2016), é perceptível o não conhecimento dos homens sobre a temática, em acrescimento da inexistência de educação permanente nos serviços, o que acaba levando a esse défice na assistência prestada aos homens, no qual acabam tendo limitações para compreender as suas reais necessidades e interferir sobre as mesmas.

Esse desconhecimento relacionado aos programas de saúde, faz com que esse grupo vulnerável não busquem os cuidados preventivos, mesmo sabendo das suas necessidades e que estão expostos a diversas patologias, classificando seus cuidados a saúde como algo que está regular, como é observado a seguir:

E1: Regular, eu preciso melhorar, preciso encaixar na rotina, como eu falei faz dois anos que eu fiz um 'checap', eu preciso colocar ele anual ou semestral, para poder ter a prevenção e não cuidar só quando tiver aí com a doença...

E3: Eu me avalio como regular, eu estava no nível bom e ai eu baixei porque, é.... a alimentação piorou, o exercício piorou, então eu me avalio como regular [...] 
E4: eu também, eи me classifico regular, antes era bom eu tinha atividades físicas, fazia dieta, enfim, eu tinha uma vida que eu sabia, eu sei que hoje eu não to na vida que eu estava antes, então eu classifico como regular, mesmo eu sabendo que eu tenho que realmente melhorar.

De acordo com Sousa et al. (2016), a cura da doença é conhecida pelo homem como a singular opção de cuidar da saúde, descartando possibilidades de promoção e prevenção da doença, com isso a busca tardia por serviços de atenção básica é fundamentada no entendimento do modelo influente de que saúde é sinônimo de ausência de doença.

Com tudo se torna necessário adquiri diferentes formas de pensar, quebrando crenças e valores para incorporar novos conceitos pertencentes a saúde masculina, incentivando a procura do homem ao serviço de saúde que deve ser ofertada de forma diferente, para garantir que o homem crie o habito dos cuidados, utilizando diferentes meios para alcança-los através de ações de educação em prol da saúde.

Independente der raça, gênero ou idade a atenção primaria será o principal contado dos pacientes, em relação a desconstrução dos preconceitos o homem necessita da pratica das políticas de saúde que tem por objetivo a promoção e prevenção de uma atenção básica mais atuante.

\section{Considerações Finais}

Os participantes demostraram que questões socioculturais, a respeito a masculinidade se fazem presentes em suas vidas, pois, relataram que por questões de cultura não procuram os serviços de saúde como forma de se prevenir, e só vão em busca de atendimento quando já "não estão mais aguentando", isto quer dizer que a dor se encontra insuportável e os cuidados em casa já não estão dando mais resultado. Sendo assim, a doença já se encontra instalada e na maioria das vezes em estágios avançado, desta forma, acabam indo para os serviços de média e alta complexidade devido à gravidade do problema.

Entretanto, os assuntos a respeito do autocuidado e a não procura por atendimentos na Unidades Básica de Saúde UBS, estão diretamente ligados, uma vez que, serve de justificativa para à não procura dos serviços na atenção primaria. Como foi abordado anteriormente no ambiente de trabalho, o cuidado com a saúde não é visto como uma pratica masculina, todavia, são uma série de fatores resistentes que estão relacionado para a não busca as unidades de saúde por parte dos homens, como o horário de atendimento, o medo de descobrir doenças e a figura feminina sempre presente, tanto na demanda da procura por atendimento, como também na formação da equipe prestadora da assistência.

Ao mesmo tempo em que os homens não procuram a unidades de atenção primaria para atendimento, os mesmos acabam ficando desprovido de informações sobre os programas existente, uma vez que, é na atenção básica que os programas de saúde são apresentados para a população através da educação em saúde. No entanto, vale ressaltar que mesmo sendo criada no ano de 2009 a Política Nacional de Atenção Integrada a Saúde do Homem - PNAISH, pouco são os homens que sabe sobre ela, qual é o seu propósito e quais foram os benefícios que sua criação trouxe para a saúde da população masculina.

Assim sendo, o desconhecimento de programas e ações voltadas para saúde do homem, fazem com que eles não procurem os cuidados preventivo, como pode ser visto, quando falado a respeito do novembro azul, que muitos só enxergavam o mês voltado para a campanha do câncer de próstata, restringindo-se assim a um único tema, quando o propósito do mês não possui apenas um objeto, mesmo que seu foco seja maior para prevenção do câncer.

Com tudo, se torna necessário que os profissionais adquiram meios no quais possa ser utilizado, para conseguir atingir o público masculino, para assim os menos se veja protagonista de sua própria saúde, e a partir daí comece a possuir conhecimento e busca os serviços a eles oferecido.

Diante do exposto sugere-se estudos direcionados acerca de estratégias de atendimento para ampliação e alcance dessa 
Research, Society and Development, v. 10, n. 3, e11010313305, 2021

(CC BY 4.0) | ISSN 2525-3409 | DOI: http://dx.doi.org/10.33448/rsd-v10i3.13305

população, a fim de fortalecer as políticas nacionais de atenção à saúde do homem.

\section{Referências}

Bardin, L. (2009). Análise de conteúdo. 70. ed. Lisboa.

Barreto, M. S., Arruda, G. O. \& Marcon, S. S. (2017). Como os homens adultos utilizam e avaliam os serviços de saúde. Revista Eletrônica de Enfermagem. Paraná, 19(3), 1-8. https://www.fen.ufg.br/revista/v17/n3/pdf/v17n3a20.pdf.

Bertolini, D. N. P., \& Simonetti, J. P. (2014). O gênero masculino e os cuidados de saúde: a experiência de homens de um centro de saúde. Escola Anna Nery Revista de Enfermagem. 18(4), 722-727. http://eean.edu.br/detalhe_artigo.asp?id=1258.

Brasil. Conselho Nacional de Saúde. Resolução $n^{o} 466$, de 12 de dezembro de $2012 . \quad$ Brasília, $2012 . \quad$ Disponível em: http://conselho.saude.gov.br/resolucoes/2012/Reso466.pdf.

Brasil. Ministério da Saúde. (2009). Política Nacional de Atenção Integral à Saúde do Homem: Princípios e Diretrizes - Brasília: Ministério da Saúde.

Cavalcanti, J. R. D., Ferreira, J. A., Henriques, A. H. B., Morais, G. S. N., Trigueiro, J. V. S., \& Torquato, I. M. B. (2014). Assistência Integral a Saúde do Homem: necessidades, obstáculos e estratégias de enfrentamento. Escola Anna Nery, 18(4), 628-634. https://doi.org/10.5935/1414-8145.20140089.

Cordeiro, S. V. L., Fontes, W. D., Fonsêca, R. L. S., Barboza, T. M., \& Cordeiro, C. A. (2014). Atenção básica à saúde masculina: possibilidades e limites no atendimento noturno. Escola Anna Nery, 18(4), 644-649. https://doi.org/10.5935/1414-8145.20140091.

Lopes, G. S. D. P., Sardagna, M. C., \& Lervolino, S. A. (2017). Motivos que levam os homens a procurar um serviço de pronto atendimento. Rev. Enfermagem Revista. Minas Gerais, 20(2), 151-155.

Modena, C. M. (2013). Os Homens E O Adoecimento Por Câncer: Um Olhar Sobre A Produção Científica Brasileira. Revista Baiana de Saúde Pública. Bahia, 37(3), 644-660. http://rbsp.sesab.ba.gov.br/index.php/rbsp/article/view/443/835.

Moreira, M. A., \& Carvalho, C. N. (2016). Atenção integral à saúde do homem: estratégias utilizadas por enfermeiras(os) nas unidades de saúde da família do interior da Bahia. Saúde \& Transformação Social. 7(3), 121-132. http://incubadora.periodicos.ufsc.br/index.php/saudeetransformacao/article/view/3660.

Oliveira, C. C., Sousa, M. A., Lira, S. V. G., Moreira, D. P., \& Pereira, A. S. (2013). A procura do homem ao serviço de saúde da atenção básica. In: Seminário nacional de pesquisa em enfermagem, natal, 17(1), 2717-2719. http://www.abeneventos.com.br/anais_senpe/17senpe/pdf/1763po.pdf.

Silva, B. T. O., Freitas, M. M., Souza, G. B. S., Hardman, M. N., Sobral, H. C. F., \& Silva, A. M. L. (2013). Promoção e prevenção da saúde do homem. Interfaces Científicas - Saúde e Ambiente. Aracaju, 2(1), 95-101. https://doi.org/10.17564/2316-3798.2013v2n1p95-101.

Silva, M. G. L. (2015). Saúde do Homem no Âmbito da Assistência de Enfermagem. In: Congresso Brasileiro dos Conselhos de Enfermagem, ed.18. João Pessoa. Anais, COFEN. http://apps.cofen.gov.br/cbcenf/sistemainscricoes/arquivosTrabalhos/I65535.E13.T12361.D9AP.pdf.

Silva, P. A. S., Furtado, M. S., Guilhon, A. B., Souza, N. V. D. O., \& David, H. M. S. L. (2012). A saúde do homem na visão dos enfermeiros de uma unidade básica de saúde. Escola Anna Nery, 16(3), 561-568. https://doi.org/10.1590/S1414-81452012000300019.

Souza, A. R., Queiroz, A. M., Florencio, R. M. S., Portela, P. P., Fernandes, J. D., \& Pereira, A. (2016). Homens nos serviços de atenção básica à saúde: repercussões da construção social das masculinidades. Revista Baiana de Enfermagem. Salvador, 30(3), 1-10. https://portalseer.ufba.br/index.php/enfermagem/article/view/16054/pdf_76.

Vieira, K. L. D., Gomes, V. L. O., Borba, M. R., \& Costa, C. F. S. (2013). Atendimento da população masculina em unidade básica saúde da família: motivos para a (não) procura. Esc. Anna Nery, Rio de Janeiro, 17(1), 120-127. http://www.scielo.br/pdf/ean/v17n1/17.pdf. 